\title{
Significance of Expression of Cyclin D as an Early Indicator in Dysplastic Transformation of Oral Mucosa in Tobacco Users
}

\author{
Sarwat Batool ${ }^{1}$ \\ Amin Fahim ${ }^{2}$ \\ Aneela Qureshi ${ }^{3}$ \\ Sumayya Anas ${ }^{4}$ \\ Nazia Qamar ${ }^{5}$ \\ Shahid Kamran 6
}

\author{
BDS, M.Phil \\ MBBS, M.Phil, PhD \\ MBBS, M.Phil, PhD \\ MSc, M.Phil \\ MBBS, M.Phil \\ MBBS, DMJ
}

OBJECTIVES: To observe the expression of Cyclin D in transition of normal oral mucosa to dysplastic lesions and to find out the possible association of immunostaining in normal oral mucosa and different grades of oral dysplasia METHODOLOGY: In this cross sectional analytical study, total of 120 diagnosed paraffin embedded blocks were included comprising of 60 samples of normal oral mucosa (Group 1) and 60 cases of various grades of oral epithelial dysplastic lesions (Group 2). Patient's record files were reviewed for age, gender and tobacco habits. Immunohistochemistry was performed by using Cyclin D monoclonal antibodies on all the tissue samples. Staining with Cyclin D was observed in each of the cases to find out their possible association as early indicator of transition from normal mucosa to oral dysplasia.

RESULTS: In Group 1, 45/60 (75\%) patients were negative for Cyclin D. In Group 2, 40/60 (66\%) were negative for Cyclin D. We found non significant association for Cyclin D staining in transition of normal oral mucosa to low grade lesions. But significant association was found in Cyclin D positivity in transition from normal mucosa to high grade dysplastic lesions. CONCLUSION: We found no association of Cyclin D as diagnostic marker between normal and early dysplastic lesions, but the expression for Cyclin D was shown to be increased with increasing irreversible grades of dysplasia ie: from normal oral mucosa to severe dysplasia.

KEY WORDS: Cyclin D, Normal oral mucosa, Oral lesions, High grade lesions, Low grade lesions.

HOW TO CITE: Batool S, Fahim A, Qureshi A, Anas S, Qamar N, Kamran S. Significance of expression of Cyclin D as an early indicator in dysplastic transformation of oral mucosa in tobacco users. J Pak Dent Assoc 2019;28(4):171-175.

DOI: https://doi.org/10.25301/JPDA.284.171

Received: 24 May 2019, Accepted: 05 August 2019

\section{INTRODUCTION}

$\mathrm{M}$ ost common malignancy found in oral cavity is oral squamous cell carcinoma (OSCC) forming about $3 \%$ of total head and neck neoplasm. ${ }^{1}$ They usually follow a premalignant progenitor termed as oral epithelial dysplastic lesions (OED) or oral potentially malignant diseases (OPMD). ${ }^{2}$ These include red and white

1. Senior Lecturer, Department of Pathology, Al Tibri Medical College Isra University Karachi Campus.

2. Associate Professor, Department of Pathology, Isra University Hyderabad.

3. Professor HOD, Department of Pathology, Al Tibri Medical College Isra University Karachi Campus.

4. Assistant Professor, Department of Pathology, Al Tibri Medical College Isra University Karachi Campus.

5. Assistant Professor, Department of Pathology, Al Tibri Medical College Isra University Karachi Campus.

6. Assistant Professor, Department of Forensic Medicine, Al Tibri Medical College Isra University Karachi Campus.

Corresponding author: “Dr. Amin Fahim” < draminfahim@gmail.com > lesions such as erythro-leukoplakias, oral submucus fibrosis, actinic cheilitis, chronic candidiasis and not the least oral lichen planus (OLP).1It is documented that OED have malignant transformation rate of about $0-20 \% .{ }^{3}$ Because of high prevalence of OSCC, these must be diagnosed before they progress to carcinoma in-situ or invasive carcinoma as suspected dysplastic lesions are usually associated with history of prolonged tobacco use, betel nut and areca nut chewing, alcohol intake, chronic irritation and oral infections. ${ }^{4}$ If OED associated with these chronic irritations are screened and diagnosed in reversible stage, It would definitely encourage the patient to change their lifestyle and eating habits so that oral lesion could be managed without progressing to irreversible OSCC. ${ }^{5}$ Suspected oral lesions are evaluated histologically for identification of dysplasia. According to pathologists, dysplasia is graded into three types based on the level of differentiation. Low grade 
dysplasia rarely leads to cancer. Moderate grade dysplasia involves the epithelial layers up to the two third of its thickness and are considered at borderline. High grade dysplasia is a type of early cancer that has high risk of becoming malignant but has not yet spread and is isolated within the basement membrane. ${ }^{5,6}$ Although routine hematoxylin and eosin staining gives sufficient idea of dysplasia grading, but immunohistochemistry is being widely used to observe more accurate cellular details as well as intracellular changes. Immunohistochemistry (IHC) is helpful in categorizing the tumor type, its progress and it shows the therapeutic usefulness. Immunoreactivity pattern with nuclear or cytoplasmic components plays a key role in investigating the early location of cellular disturbance. ${ }^{7}$ Among different immunomarkers being widely used for OSCC, Cyclin D is focused by researchers which has proven its role in carcinogenesis. It acts as cell cycle regulator. ${ }^{8}$ Variety of cyclins are being used for diagnostic as well as prognostic marker. Among them Cyclin D is a key regulator of G1-S phase of cell cycle and it's activation is controlled by Cyclin dependent kinase inhibitors (CDKI). ${ }^{8}$ Cyclin D has been shown to get amplified in B cell lymphomas and oral cavity cancers. ${ }^{9}$ According to literature there is strong evidence proving advanced OSCCis a result of disturbed amplification of Cyclin D which causes shortened G1-S phase and uncontrollable cell growth. ${ }^{9} 10$ However little is known for the overexpression of Cyclin D in oral dysplastic lesions and its role as anticancer drug by blocking cellular proliferation. ${ }^{10,11}$ Early diagnosis of oral dysplastic lesion is an important step in abstaining tobacco chewing habit and can improve patient survival. Therefore this study began with the aim to observe if there is any altered expression of Cyclin D in transition of normal oral mucosa to dysplastic oral lesions in tobacco users.

\section{METHODOLOGY}

This was a cross sectional analytical study, which started after approval from institutional ethical review committee and was done in Department of Pathology Al-Tibri Medical College and Hospital, Karachi and Department of Histopathology Dow University of Health Sciences, Karachi. This study started in March 2018 and was ended in November 2018. The samples were collected by nonprobability convenient sampling technique. Samples were grouped as 60 diagnosed samples of normal oral mucosa as group 1 and 60 diagnosed cases of different grades of OED lesions as group 2. All the grades of group 2 were further labelled as $\mathrm{a}, \mathrm{b}$ and $\mathrm{c}$ according to increasing grade of dysplasia i.e. mild dysplasia as Group 2a $(\mathrm{n}=20)$, moderate dysplasia as Group 2b $(n=21)$ and severe dysplasia as Group 2c $(n=19)$.
Biopsy specimen from individuals who received radiotherapy or chemotherapy were excluded from the study. A total of 120 diagnosed paraffin embedded blocks were retrieved from histopathology laboratory of Dow University of Health Sciences Karachi. Institutional permission and approval was taken to use paraffin embedded histopathological blocks for research purpose. The study material was taken from biopsies received from patients who were treated between the year 2010 to 2017 and their medical records were studied for confirmation of tobacco usage. Histopathological grading was done by using H\&E staining by experienced pathologist for reconfirmation for various grades of group 2 .

For IHC 5microns thick sections were cut from each of the blocks. Manual's instructions were followed for IHC protocol. The validity of immunohistochemistry staining was provided by using positive controls which was B cell lymphoma for Cyclin D. Negative controls were taken by omitting primary antibody. The antibodies used was Anti Cyclin D (clone P2D11F11, RTU-CYCLIN-GM, dilution 1:100 Dakocytomotion).

Qualitative method which depended upon subjective perception and judgment was chosen for evaluation for immunoreactivity. Stained slides were observed using light microscope under $\mathrm{x} 40$ for evaluation of stained area and under x100 for evaluating intensity of staining. Random 10 fields were selected to visualize positive and negative stained cells. To avoid misleading interpretations, only homogenous staining was evaluated. For heterogeneous staining, dominant pattern of staining was chosen. Any light yellow to dark brown nuclear staining of at least $10 \%$ of focused area was considered as positive. For negative staining showing background stain only or barely visible intra nuclear brown staining in $<10 \%$ area was considered.

SPSS IBM version 20 was used for data entry and analysis. Qualitative variables were calculated as frequencies and percentages. Chi-squared ( 2 ) test was used to compare qualitative variables. For quantitative variable like age mean and standard deviation were calculated. The $\mathrm{p}$ value $\leq 0.05$ was considered as significant.

\section{RESULTS}

Demographical variables with frequencies and percentages are summarized in Table-1. All of the oral dysplastic cases were tobacco users of varying frequency. Most of the mild and moderate oral dysplastic cases belonged to regular tobacco users i.e. 34/41(83\%) and rest of them were occasional tobacco users i.e. 7/41 (17\%). However all of the severe dysplastic cases were regular tobacco users. We also found positive association of degree of dysplasia with tobacco history ( $\mathrm{p}=0.047$ ). Among group 1 , we found 
Table 1: Demographic features as frequencies and percentages among study participants

\begin{tabular}{|c|c|c|c|}
\hline \multicolumn{2}{|c|}{ Demographical variables } & \multicolumn{2}{|c|}{ Type of oral mucosa } \\
\hline & & $\begin{array}{l}\text { Normal oral mucosa } \\
\text { (Group1) }(n=60)\end{array}$ & $\begin{array}{l}\text { Dysplastic mucosa } \\
\text { (Group 2) }(n=60)\end{array}$ \\
\hline \multirow[t]{2}{*}{ Gender } & Male & $33(55 \%)$ & $48(80 \%)$ \\
\hline & \begin{tabular}{|l|} 
Female \\
\end{tabular} & $27(45 \%)$ & $12(20 \%)$ \\
\hline \multirow[t]{2}{*}{ Age } & $<40$ years & $30(50 \%)$ & $15(25 \%)$ \\
\hline & $>40$ years & $30(50 \%)$ & $45(75 \%)$ \\
\hline \multirow{2}{*}{$\begin{array}{l}\text { Tobacco } \\
\text { habits }\end{array}$} & Regular & $50(83 \%)$ & $53(88 \%)$ \\
\hline & Occasional & $10(16 \%)$ & $7(12 \%)$ \\
\hline \multicolumn{4}{|c|}{ M:F in Group 1: 1.2:1 } \\
\hline \multicolumn{4}{|c|}{$M: F$ in Group 2: 4:1 } \\
\hline \multicolumn{4}{|c|}{ Group 1: Mean $\pm S D$ for age $42 \pm 9$ year } \\
\hline \multicolumn{4}{|c|}{ Group 2: Mean \pm SD for age $45 \pm 5$ year } \\
\hline
\end{tabular}

Figure 1: Photomicrographs showing oral epithelium with Cyclin D immunostaining (x100).

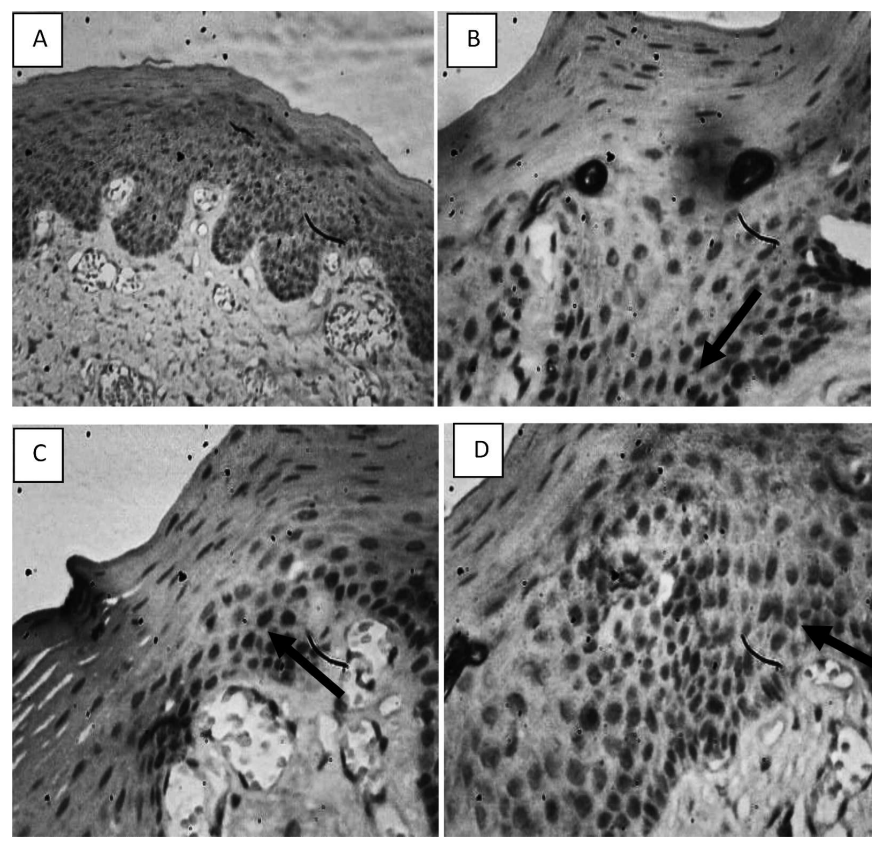

A: Shows negative Cyclin D expression in normal oral mucosa (Cyclin D immunostaining). B: $(\longrightarrow)$ Shows presence of mild brown nuclear staining of Cyclin D on basal layer of mild dysplastic epithelium. C: $(\longrightarrow)$ Shows presence of mild brown nuclear staining of Cyclin D on basal layer of moderate dysplastic epithelium. D: $(\longrightarrow)$ Shows presence of mild brown nuclear staining of Cyclin D on basal and supra basal layer of severe dysplastic epithelium majority of samples were negative for Cyclin D. Similarly in group 2, most of samples were negative for Cyclin D. These frequencies and percentages are shown in Table-2 and illustrated in Figure-1. According to Table-3, we further found non-significant association for Cyclin D staining in
Table 2: Expression of Cyclin D scores in various study groups as percentages and frequencies

\begin{tabular}{|c|c|c|}
\hline \multirow{2}{*}{ Groups } & \multicolumn{2}{|c|}{ Staining expression } \\
\cline { 2 - 3 } & $45(75 \%)$ & Positive \\
\hline Group 1 $(n=60)$ & $40(67 \%)$ & $20(33 \%)$ \\
\hline Group 2 $(n=60)$ & $18(90 \%)$ & $2(10 \%)$ \\
\hline Group 2a(n=20) & $14(67 \%)$ & $7(33 \%)$ \\
\hline Group 2b(n=21) & $8(42 \%)$ & $11(58 \%)$ \\
\hline Group 2c(n=19) & $\begin{array}{l}\text { Group 1: Normal oral mucosa } \\
\text { Group 2: Oral dysplastic lesions } \\
\text { Group 2a: Mild dysplasia } \\
\text { Group 2b: Moderate dysplasia } \\
\text { Group 2c: Severe dysplasia }\end{array}$ \\
\hline
\end{tabular}

Table 3: Association of Cyclin D staining scores between Group 1, Group 2 using Chi square test

\begin{tabular}{|l|c|c|c|c|}
\hline \multirow{2}{*}{ Groups } & \multicolumn{2}{|c|}{ Staining scores } & \multirow{2}{*}{ Total } & \multirow{2}{*}{ P value } \\
\cline { 2 - 3 } & Negative & Positive & & \\
\cline { 2 - 3 } Group 1 & 45 & 15 & 60 & \multirow{2}{*}{0.315} \\
\hline Group 2 & 40 & 20 & 60 & \\
\hline Group 1 & 45 & 15 & 60 & \multirow{2}{*}{0.156} \\
\hline Group 2a & 18 & 2 & 20 & \\
\hline Group 1 & 45 & 15 & 60 & \multirow{2}{*}{$0.008^{*}$} \\
\hline Group 2b & 14 & 7 & 21 & \\
\hline Group 1 & 45 & 15 & 19 & \\
\hline Group 2c & 8 & 11 & 60 & \\
\hline
\end{tabular}

Group 1: Normal oral mucosa

Group 2: Oral dysplastic lesions

Group 2a: Mild dysplasia

Group 2b: Moderate dysplasia

Group 2c: Severe dysplasia

transition from normal oral mucosa to early dysplastic lesions. However significant association was seen for Cyclin D staining in transition of normal mucosa to high dysplastic lesions.

\section{DISCUSSION}

Various studies have been done on oral epithelial dysplasia with the objective of knowing diagnosis and prognosis. In our study $80 \%$ oral dysplastic samples belonged to males with M:F 4:1. Similar results were shown in the study of Juneja S in 2015 which showed $53 \%$ of their dysplastic 
samples belonged to males. ${ }^{12}$ However, in contrast Jaber M in 2010, showed that oral dysplasia was common in both male and females equally with M:F $1: 1{ }^{13}$ This might be due to larger sample size and westernized environment where their study was conducted. In our study most of the oral dysplastic cases were distributed in middle aged patients with mean age $45 \pm 5$ years. Similar results were shown in study done Ho PS in 2009 which showed predominance of oral dysplasia in middle aged patients. This similarity might be due to similar sample size with similar gender distribution in their study. ${ }^{14}$ Gopinath D et al in 2016 showed evidence of oral dysplasia in individuals with mean age $51 \pm 17$ years in males and $56 \pm 12$ years in females..$^{15}$ A study done in 2014 by Starzynska A et al found dysplastic lesions were most commonly found in patients with age range of 51-60 years. ${ }^{16}$ In this study strong history of chewing tobacco was seen in $88 \%$ in oral dysplastic cases with $12 \%$ cases from occasional tobacco users but nonsignificant association was shown in tobacco use and grade of oral dysplasia $(\mathrm{p}=0.136)$. These results were in concordance with the findings of Shetty P in 2017 showing evidence of dysplasia in individuals with low to moderate tobacco consumption. ${ }^{17}$ Similar findings were reported by Mohiuddin S in 2015 showing positive association of dysplasia with smokeless tobacco and areca nut. ${ }^{18}$ The present study showed $75 \%$ cases with negative scores for Cyclin D and 25\% samples showing mild staining in normal oral mucosa. It has been previously shown in studies done by Bogozi B in 2012, Mishra R in 2009 that Cyclin D is totally negatively expressed in normal oral mucosa. ${ }^{19,20}$ However they used smaller sample size and all normal oral mucosal samples were strictly recruited from tobacco users. In contrast to our results, study done by Ramakrishna A in 2013 showed $40 \%$ of normal oral mucosal samples with moderate staining but the sample size was smaller than the present study. ${ }^{21}$ Our study showed $67 \%$ oral dysplastic lesions negative for Cyclin D which was in similarity with study done by Lam KY and colleagues in 2000 proved faint expression of Cyclin D is only limited to few high grade oral lesions and all of the OSCC cases. He further commented that this finding was because of shorter half life of Cyclin D during G1-S phase of cell cycle. ${ }^{22}$ This finding was further reinforced by the evidences provided by Mishra R in 2009 showing no immunoreactivity with Cyclin $\mathrm{D}$ in normal and potentially malignant lesions..$^{20}$ Our study showed nonsignificant association of Cyclin D expression in various grades of oral dysplasia which was reinforced by the results of Turrati E in 2005. According to him Cyclin D was totally negative in low grade dysplasia and was positive in only $8 \%$ of high grade dysplasia. ${ }^{23}$ In present study Cyclin D expression was reported in $33 \%$ of dysplastic lesions and significant association $(\mathrm{p}=0.08)$ was observed in transition of normal mucosa to severe dysplasia which coincided with previous study done by Ramasubramanian A et al in 2013 showing Cyclin D positivity for dysplastic lesions and its strong expression was favored for irreversible grades of dysplastic lesions $(\mathrm{p}=0.001) .{ }^{24}$ Our study revealed $90 \%$ cases of mild dysplasia with negative expression of Cyclin $\mathrm{D}$, moderate dysplastic cases showed $66 \%$ cases with negative expression for Cyclin D and 33\% cases showed mild expression. Similarly severe dysplastic lesions showed negative expression for Cyclin D in $42 \%$ cases as well as positive expression was observed in 58\% cases.In 2017 Patel SB and colleagues investigated all of the mild dysplasia cases with mild expression of Cyclin D. Moderate dysplastic cases showed $66 \%$ of positivity. Similarly severe dysplasia showed positive expression in $92 \%$ cases..$^{25}$ These dissimilarities might be due to smaller sample sizes used in their studies.

\section{CONCLUSION}

Oral squamous cell carcinoma treatment in its late presentation, end up with high rates of mortalities and diminished chances of survival. It is not surprising that early oral epithelial dysplastic lesions are curable. Therefore it is a real need to imply methods for early detection of oral dysplastic lesions especially in those with risk factors like tobacco use. Cyclin D showed non-significant association in transition from normal mucosa to early dysplastic cases and hence it was not found as reliable diagnostic marker in early oral dysplastic lesions but strong and significant amplification of Cyclin D was seen in transition from normal oral mucosa to severe dysplasia which showed its usefulness as diagnostic tool in high grade dysplastic lesions.

\section{CONFLICT OF INTEREST}

None declared

\section{REFERENCES}

1. Yardimci G, Kutlubay Z. Precancerous Lesions of Oral Mucosa. World J Clin Cases 2014;2:866-72.

https://doi.org/10.12998/wjcc.v2.i12.866

2. Tanaka T, Tanaka M, Tanaka T. Oral Carcinogenesis and Oral Cancer Chemoprevention: A Review. Path Res Int.2011.

https://doi.org/10.4061/2011/431246

3. Gopinath D, Thannikunnath BV, Neermunda SF. Prevalence of Carcinomatous Foci in Oral Leukoplakia: A Clinicopathologic Study of 546 Indian Samples. J ClinDiagn Res 2016;10:78-83.

https://doi.org/10.7860/JCDR/2016/16815.8305

4. Richards D. Oral Leukoplakia: Malignant transformation rate is 
highly variable. The dental elf. [updated on 15th Jul 2018; cited om 4th Aug 2018]; Available from: https://www.nationalelfservice.net/ dentistry/oral-medicine-and-pathology/oral-leukoplakia-malignanttransformation-rate-highly-variable/

5. Neville BW, Day TA. Oral Cancer and Precancerous Lesions. Can J Clin 2002;52:195-15.

https://doi.org/10.3322/canjclin.52.4.195

6. Geetha KM. Grading of Oral Epithelial Dysplasia: Points to Ponder. J Oral Maxillofac Path 2015;19:198-204.

https://doi.org/10.4103/0973-029X.164533

7. Devi M, Vijayalakshmi D, Dhivya K, Vezhavendhan N. A study on awareness of early detection and screening of potentially malignant oral disorders and oral cancer. J NTR Univ Health Sci. 2017;6:35-8

8. Inoue K, Fry EA. Aberrant Expression of cyclin d1 in cancer. Signal Transduction Insights. 2015;1-13.

https://doi.org/10.4137/STI.S30306

9. Shan YS, Hsu HP, Lai MD, Hung YS, Wang CY, Yen MC et al. Cyclin D1 overexpression correlates with poor tumor differentiation and prognosis in gastric cancer. Oncol Lett. 2017;14:4517-526. https://doi.org/10.3892/ol.2017.6736

10. Bakr MM, Guan S, Firth R, Love RM. Cyclin D and P27KIP1: Gate keepers of dysplasia. J Immunol Sci. 2018;

11. Balakrishnan A, Vyas A, Deshpande K, Vyas D. Pharmacological cyclin dependent kinase inhibitors: Implications for colorectal cancer. World J Gastroenterol. 2016;22:2159-164.

https://doi.org/10.3748/wjg.v22.i7.2159

12. Juneja S, Chaitanya NB, Agarwal M. Immunohistochemical expression of Bcl-2 in oral epithelial dysplasia and oral squamous cell carcinoma. Indian J Cancer. 2015;52:505-10.

https://doi.org/10.4103/0019-509X.178411

13. Jaber MA. Oral epithelial dysplasia in non-users of tobacco and alcohol: an analysis of clinicopathologic characteristics and treatment outcome. J Oral Sci. 2010;52:13-21.

https://doi.org/10.2334/josnusd.52.13

14. Ho P, Chen PL, Warnakulasuriya S, Shieh TH, Chen YK, Huang IY. Malignant transformation of oral potentially malignant disorders in males: a retrospective cohort study. BMC Can. 2009;9(260). https://doi.org/10.1186/1471-2407-9-260

15. Gopinath D, Thannikunnath BV, Neermunda SF. Prevalence of Carcinomatous Foci in Oral Leukoplakia: A Clinicopathologic Study of 546 Indian Samples. J ClinDiagn Res 2016;10:78-83.

https://doi.org/10.7860/JCDR/2016/16815.8305
16. Starzynska A, Pawlowska A, Renkielska D, Michajlowski I, Sobjanek M, Blazewicz I. Oral premalignant lesions: epidemiological and clinical analysis in the northern Polish population. PostepyDermatolAlergol. 2014;31:341-50.

https://doi.org/10.5114/pdia.2014.40932

17. Shetty P, Khargekar NC, Debnath A, Khargekar NR, Srivastava BK, Hakeen NEF. Determinants of Tobacco Use and Prevalence of Oral Precancerous Lesions in Cab Drivers in Bengaluru City, India. Int J Prev Med. 2017;8.

https://doi.org/10.4103/ijpvm.IJPVM_225_17

18. Mohiuddin S, Fatima N, Hosein S, Fatima N. High risk of malignant transformation of oral submucous fibrosis in Pakistani females: A potential national disaster. J Pak Med Assoc. 2016;66:1362-366.

19. Bogozi B, Mezei T, Bocskey I. Expression of Cyclin D1 in Oral Leukoplakia Compared with Normal Mucosa, Benign and Malignant Tumors of the Oral Cavity. ActaMedica. 2012;58.

20. Mishra R, Das BR. Cyclin D1 expression and its possible regulation in chewing tobacco mediated oral squamous cell carcinoma progression. Archives Oral Biol. 2009;54:917-23.

https://doi.org/10.1016/j.archoralbio.2009.07.003

21. Ramakrishna A, Shreedhar B, Narayan T, Mohanty L, Shenoy S, Jamadar S. Cyclin D1 an early biomarker in oral carcinogenesis. J Oral MaxillofacPathol. 2013;17:351-57.

https://doi.org/10.4103/0973-029X.125189

22. Lam KY, Irene OL, Yuen APW, Kwong DLW, William W. Cyclin D1 expression in oral squamous cell carcinomas: Clinicopathological relevance and correlation with p53 expression. J Oral Pathol Med. 2000;29:167-72.

https://doi.org/10.1034/j.1600-0714.2000.290404.x

23. Turrati E, Neves AC, Magalhaes MHCG, Souse SOM. Assessment of c-Jun, c-Fos and Cyclin D1 in premalignant and malignant lesion. J Oral Sci. 2005;47:71-6.

https://doi.org/10.2334/josnusd.47.71

24. Ramasubramanian A, Ramani P, Sherlin HJ, Premkumar P, Natesan A, Thiruvengadam C. Immunohistochemical evaluation of oral epithelial dysplasia using cyclin-D1, p27 and p63 expression as predictors of malignant transformation. J Nat Sci, Bio Med. 2013;4:349-58. https://doi.org/10.4103/0976-9668.117011

25. Patel SB, Manjunatha BS, Shah V, Soni N, Sutariya R. Immunohistochemical evaluation of p63 and cyclin D1 in oral squamous cell carcinoma and leukoplakia. J Korean Assoc Oral Maxillofac Surg. 2017;43:324-30.

https://doi.org/10.5125/jkaoms.2017.43.5.324 